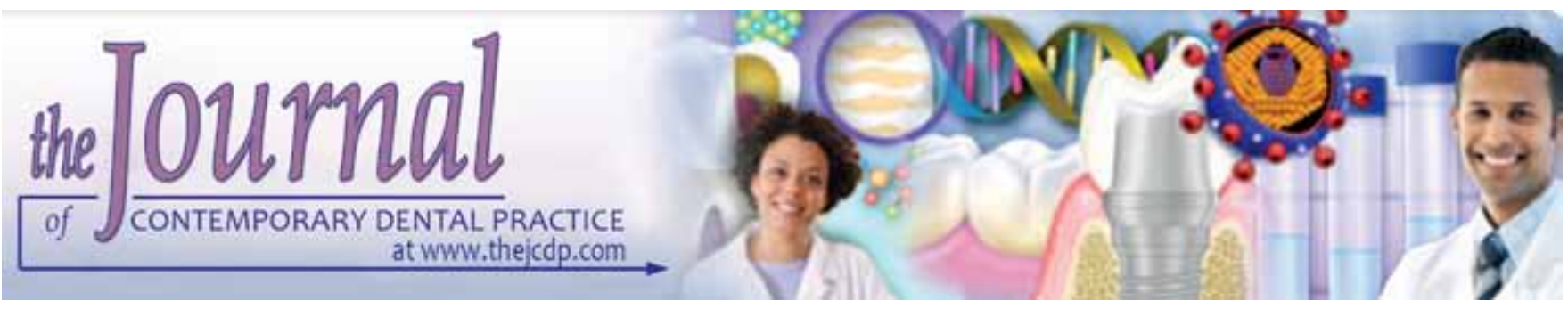

\title{
The Use of Controlled Release Locally Delivered $10 \%$ Doxycycline Hyclate Gel as an adjunct to Scaling and Root Planing in the Treatment of Chronic Periodontitis: Clinical and Microbiological Results
}

\author{
Shabeer Ahamed, Md Jalaluddin, Imran Khalid, Ninad Moon, TK Shafi, Fareedi Mukram Ali
}

\begin{abstract}
Background: Local delivery of antimicrobial agents provides higher concentration of the drug in the periodontal site for longer periods than systemically delivered methods. In the present study an attempt is made to know the efficacy of controlled local drug delivery of doxycycline as an adjunctive treatment in the management of chronic periodontitis.
\end{abstract}

Materials and methods: A total of 12 patients, diagnosed as Chronic Periodontitis in the age of 25 to 55 years, were selected of both the sexes in this study. They were divided into Experimental group consisted of 30 sites who received complete scaling and root planing (SRP) followed by placement of Atridox gel and control group consisted of 30 sites who received only SRP. Clinical parameters were recorded at, baseline, days 30, 90 and 180. Parameters were plaque index, gingival index, gingival bleeding index and microbial analysis, probing pocket depth and Clinical attachment level (CAL). The microbiological analysis was done at baseline visit and at 90th day. Probing pocket depth and CAL were recorded only on day 0 and 180th day.

Results: In 180 days study, both the groups exhibited a significant improvement in periodontal status. Significant gain in attachment level was observed in both the group. Between both the groups the clinical parameters in the experimental groups exhibited better results as compared to the control group. Both the groups exhibited significant reduction in the number of spirochetes.

Conclusion: Combination therapy of SRP and $10 \% \mathrm{DH}$ gel demonstrated better results at all levels suggesting that this therapy can play a significant role as an adjunct to SRP in the management of chronic periodontitis.

Keywords: Antimicrobial agent, Chronic periodontitis, Doxycycline hyclate (Atridox), Local drug delivery.

How to cite this article: Ahamed S, Jalaluddin Md, Khalid I, Moon N, Shafi TK, Ali FM. The Use of Controlled Release Locally Delivered $10 \%$ Doxycycline Hyclate Gel as an adjunct to Scaling and Root Planing in the Treatment of Chronic Periodontitis: Clinical and Microbiological Results. J Contemp Dent Pract 2013;14(6):1080-1086.

\section{Source of support: Nil}

Conflict of interest: None declared

\section{INTRODUCTION}

Periodontal diseases are infections caused by microorganisms that colonize the tooth structure at or below the gingival margin. The elimination or alteration of the microbial pathogens present in the subgingival plaque is the primary objective of periodontal therapy. A number of different nonsurgical and surgical therapies has been successful in achieving this goal. The primary nonsurgical approach involves mechanical scaling and root planing (SRP). An additional nonsurgical approach to periodontal treatment would be to reduce the magnitude of the inflammation by various approaches which include systemic antibiotic, local drug delivery systems and mouth washes. One such promising approach is the local delivery of antimicrobial agents in the periodontal pockets.

Doxycycline is approved as a systemic antibiotic since 1980 's and is being used as a systemically delivered antibiotic in the treatment of periodontal disease. Systemically, doxycycline has the ability to concentrate in crevicular fluid, eliminate Actinobacillus actinomycetemcomitans and demonstrates a wide spectrum of activity against other suspected periodontal pathogens. Studies have shown that systemic Doxycycline suppresses Actinobacillus actinomycetemcomitans, Porphyromonas gingivalis, Prevotella intermedia, Eikenella corrodens, Fusobacterium nucleatum and Spirochetes present in recurrent lesions of high-risk patients. ${ }^{1}$

In addition to its antibacterial action, it has also nonantibacterial action, like anticollagenolytic action, inhibition of 
bone resorption, anti-inflammatory action. More recently doxycycline has shown to be substantive to both cementum and dentin, suggesting that root surfaces could serve as substrates for the deposition and subsequent slow release of doxycycline.

Controlled release local delivery of antimicrobial agents provides higher concentration of the drug in the periodontal site for longer periods than systemically delivered methods. The concept of controlled drug delivery was developed in 1970's by Dr Max Goodson. It was based on the theory that if one could substantially improve the cellular specificity of a drug there would be an accompanying significant improvement in the therapeutic index, i.e. the ratio of efficacy to side effects. Recently, a local anti-infective treatment for periodontitis has been introduced that delivers a formulation of $10 \%$ doxycycline hyclate to the periodontal pocket in a biodegradable liquid polymer that solidifies on contact with the gingival crevicular fluid. As it is biodegradable, it delivers doxycycline at high levels to the site for 7 to 14 days.

An animal study in beagle dogs initially suggested beneficial aspects of locally delivered doxycycline. ${ }^{2}$ Studies undertaken by Garrett et $\mathrm{al}^{3}{ }^{3}$ when compared to SRP indicated that $10 \%$ doxycycline hyclate is as effective as $\mathrm{SRP}$ in reducing clinical signs of periodontitis, Walker et $\mathrm{al}^{4}$ showed that $10 \%$ doxycycline delivery system significantly reduced the anaerobic bacteria in plaque and did not change the amount of resistant bacteria. Hence in the present study an attempt is made to know the efficacy of controlled local drug delivery of doxycycline as an adjunct to SRP in the management of chronic periodontitis.

\section{MATERIALS AND METHODS}

Study populations: A total of 12 patients who were diagnosed as suffering from Chronic Periodontitis in the age of 25 to 55 years, were selected from the Out Patient Department of Periodontics, Bangalore Institute of Dental Sciences, Bengaluru. Both the sexes were included in this study. Informed consent was taken from the patients.

At prebaseline visit periodontal screening was done to assess current disease status, periodontal treatment history and medical history. Subjects diagnosed as chronic periodontitis (American Academy of Periodontology (AAP) -1999) were included. Patients must be 25 to 55 years of age and in good systemic health. Patients must have a minimum of 5 nonadjacent periodontal pockets measuring $\geq 5 \mathrm{~mm}$.

The exclusion criteria included subjects with compromised medical conditions, if they had contraindicated medications, pregnancy or active lactation, have received any chemotherapeutics during past 6 months, allergy to components of periodontal dressing, known hypersensitive to doxycycline or lignocaine and if they have received any periodontal therapy for the past 6 months of the baseline visits. Ethical clearance was obtained from the ethical committee of Bangalore Institute of Dental Sciences, Bengaluru, Karnataka, India.

\section{Treatment Procedure and Clinical Evaluations}

A parallel design was used in this study for the delivery of treatment. Thirty sites from six subjects were selected for experimental group who received SRP followed by placement of Atridox gel. The control group consisting of 30 sites from six subjects received only SRP. All the sites selected for the studywere nonadjacent and had 6 to $7 \mathrm{~mm}$ of probing depths. Before recording the clinical parameters at the baseline visit any visible supragingival plaque was collected with a sterile instrument and then the subgingival sampling was performed using sterile endodontic paper points. Microbiological sampling was done from one preselected site with deepest pocket. Endodontic paper points were placed in the periodontal pocket for 10 seconds till resistance was felt. Followed by this, these paper points were placed in the sterile pellets containing two or three drops of $0.85 \%$ sodium chloride solution. The clinical parameters included plaque index (Silness and Loe, 1964), ${ }^{5}$ bleeding index (Ainamo and Bay, 1975), ${ }^{6}$ and Gingival Index (Loe and Silness 1963), ${ }^{7}$ probing pocket depth and clinical attachment level.

The clinical parameters were evaluated at baseline visit and 30th, 90th and 180th days (Figs 1 and 3). Whereas probing pocket depth and clinical attachment level recording was done at baseline, 90th and at 180th day. The microbiological analysis was done at baseline visit and at 90th day. All periodontal measurements were made using a periodontal probe graduated in $1 \mathrm{~mm}$ increment. Reading was rounded to the nearest $\mathrm{mm}$.

After recording the clinical parameters, a through SRP was done using hand instruments and ultrasonic scalers in experimental group and control group sites.

\section{Application of Doxycycline Hyclate $10 \%$ Gel}

The two syringes of the formulations were mixed as per the instruction from the manufacturer. Once mixed, the coupled syringes were allowed to set at room temperature for 15 minutes and then mixed for another 10 cycles before use. With the help of $2 / 3$ gauge cannula attached to the delivery syringe, the above prepared product was slowly expressed into the periodontal pocket starting from the base of the pocket until it reached the gingival margin (Fig. 2). A moist curette was used to pack any overflow of material down 
into the pocket. Periodontal dressing was used to achieve retention of the product into the pocket.

\section{Postprocedure Instruction}

After the treatment procedure the patients were instructed not to brush or floss on the treated area for 7 days. Oral hygiene instructions were given to each patient to brush with modified bass technique. The patients were asked to report immediately, if the material or pack is dislodged before the scheduled recall visit or if pain, swelling or any other problems occur. Patients were recalled and at each visit oral hygiene instructions were reinforced Patients were instructed not to use mouth wash, systemic antibiotics throughout the study period. The patients were recalled after 7 days for the removal of periodontal dressing.

\section{Statistical Method}

Statistical analysis of clinical parameters was done using paired ' $t$ ' test. Statistical analysis of intra group comparison for microbiological study was done using Wilcoxon signed rank test. Statistical analysis of intergroup comparison was done using Mann-Whitney U-test.

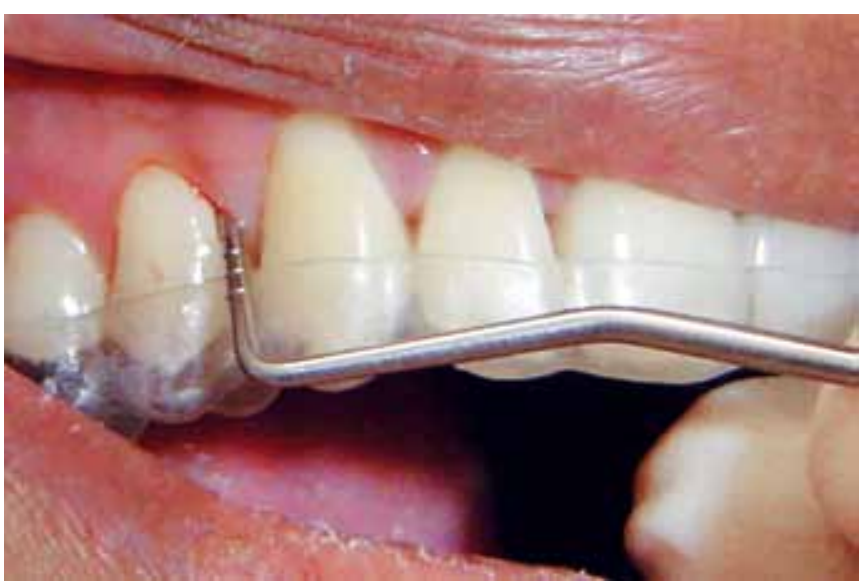

Fig. 1: Clinical measurement at baseline

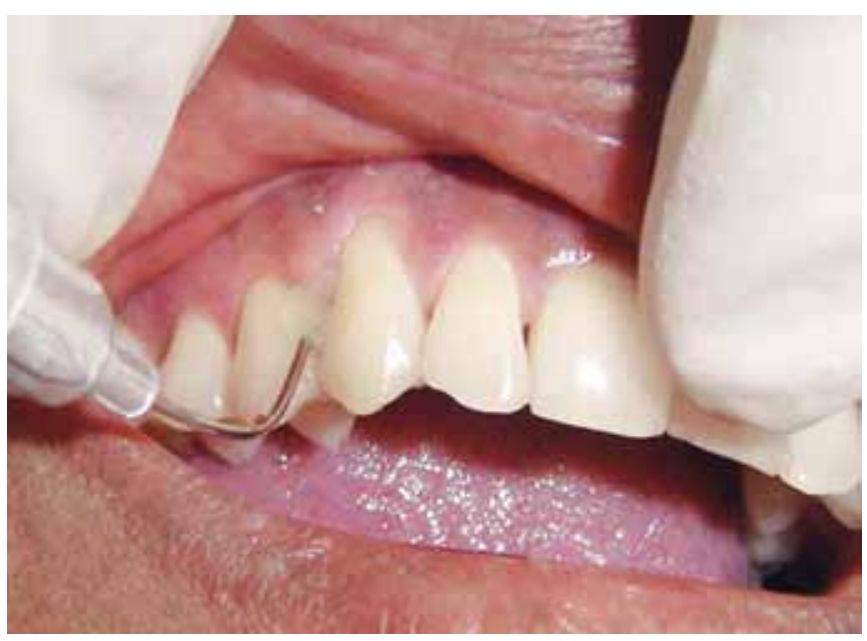

Fig. 2: Application of doxycycline hyclate $10 \%$ gel

\section{RESULTS}

Twelve patients including seven males and five females were selected for this study between age group 25 and 55 years. All the 12 patients were evaluated for the period of 180 days. No adverse effects or any complications were reported by the patients. The results of this study are presented in Graphs 1 to 6 .

\section{CLINICAL PARAMETERS}

\section{Plaque Index}

The overall plaque index scores showed significant improvement from baseline to 180 days for both experimental and control group. The mean value of overall plaque index at baseline was 1.68 for experimental group and 1.5 for control group. Mean plaque scores were reduced to 0.16 for experimental group and 0.12 for control group at 180 days respectively. Intragroup comparison showed statistically significant reduction in plaque scores for experimental as well as control group from baseline to 180 days ( $p<0.0001)$. Inter group comparisons between experimental and control group showed no statistically significant difference from baseline to 180 days $(\mathrm{p}>0.2)$.

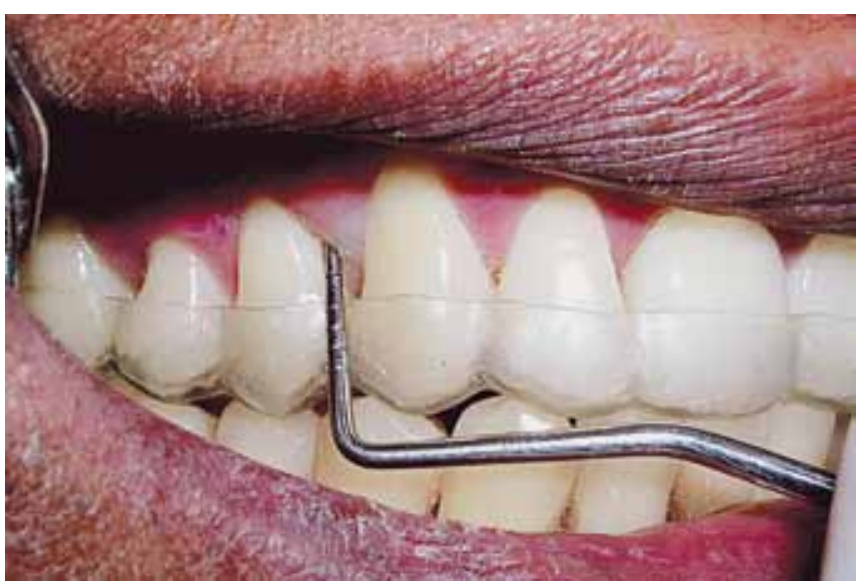

Fig. 3: Clinical measurement at 180th day

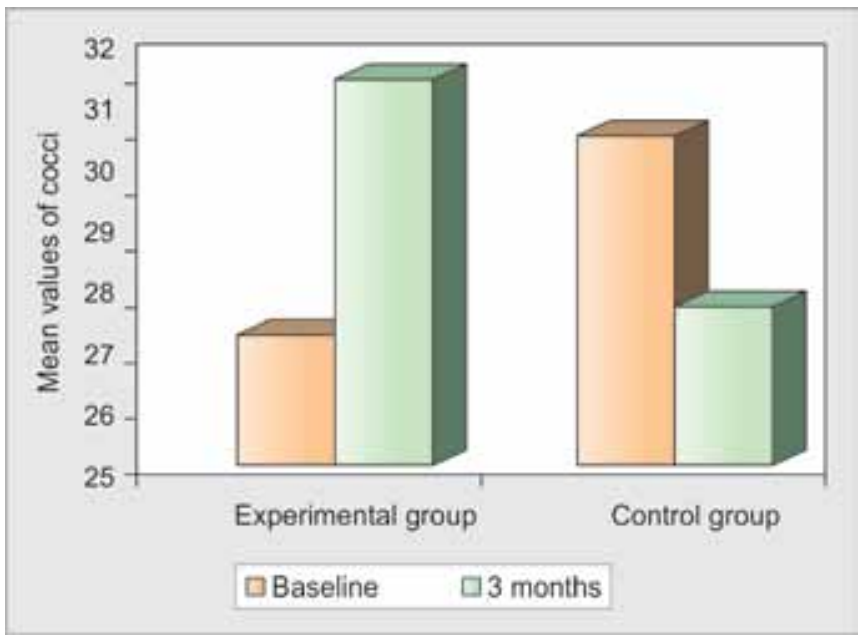

Graph 1: Mean values of cocci 


\section{Gingival Index}

The mean value of overall gingival index at baseline was 1.49 for experimental group and 1.55 for control group. Mean gingival index scores were reduced to 0.17 for experimental group and 0.29 for control group from baseline to 180 days respectively. Intragroup comparisons showed that there was statistically significant reduction in both the groups at 180th days $(\mathrm{p}<0.0002)$. When intergroup comparisons were made between experimental and control group there was no statistical significance $(\mathrm{p}>0.07)$.

\section{Bleeding on Probing}

Intragroup comparison of experimental as well control group showed reduction in percentage of bleeding scores at test sites for 180 th days from baseline. This was found to be statistically significant $(p<0.0002)$ for both experimental as well as control group. In the experimental sites percentage of bleeding scores was reduced from $100 \pm 0$ to $21.67 \pm 5.16$ at 180 days from baseline. In the control sites percentage of bleeding scores was reduced from $100 \pm 0$ to $34.17 \pm 12.41$ at 180 days from baseline.

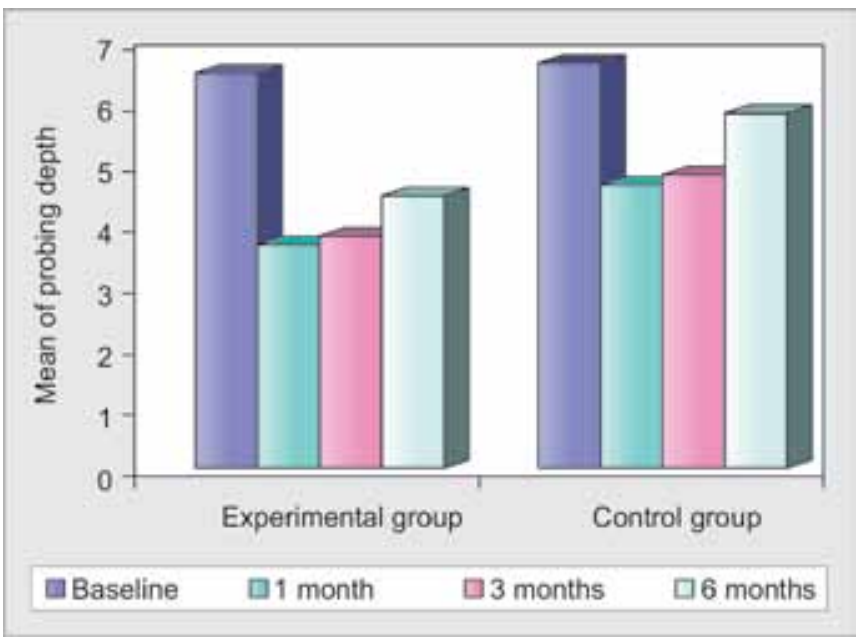

Graph 2: Mean pocket depth reduction at different time intervals

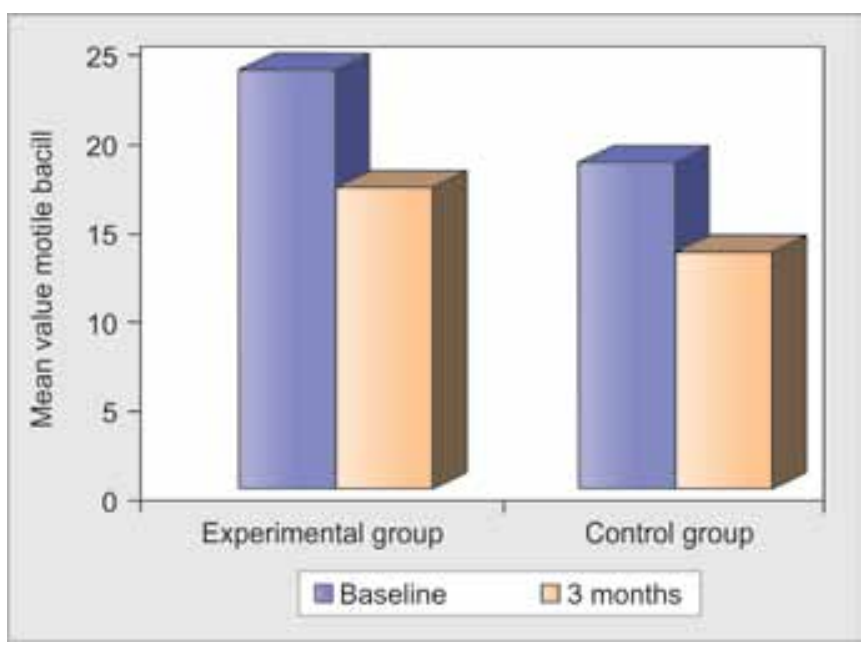

Graph 3: Mean values of motile bacilli
Intergroup comparison between experimental and control group showed statistically significant reduction in bleeding percentage scores at 180th days from baseline. This was found to be statistically significant $(\mathrm{p}<0.002)$.

\section{Periodontal Pocket Depth}

The mean probing pocket depth at baseline was $6.4 \pm 0.2 \mathrm{~mm}$ for experimental group and $6.6 \pm 0.3 \mathrm{~mm}$ for control group respectively. The mean reduction in probing pocket depth was $1.9 \mathrm{~mm}$ for experimental group and $0.8 \mathrm{~mm}$ for control group at 180 days respectively. Intragroup comparison between baseline and 180 days in the experimental sites showed there was reduction in probing depth of $4.5 \pm 1.1 \mathrm{~mm}$ which was found to be statistically 11 significant $(p<0.006)$. In the control sites there was a mean reduction in probing depth of $5.8 \pm 1.1 \mathrm{~mm}$ between baseline and 180 th days. This was found to be statistically significant $p<0.05$. When intergroup comparisons were made between experimental and control group there was statistically significant reduction in probing depth from baseline to 180th days. This was found to be statistically significant $(\mathrm{p}<0.04)$.

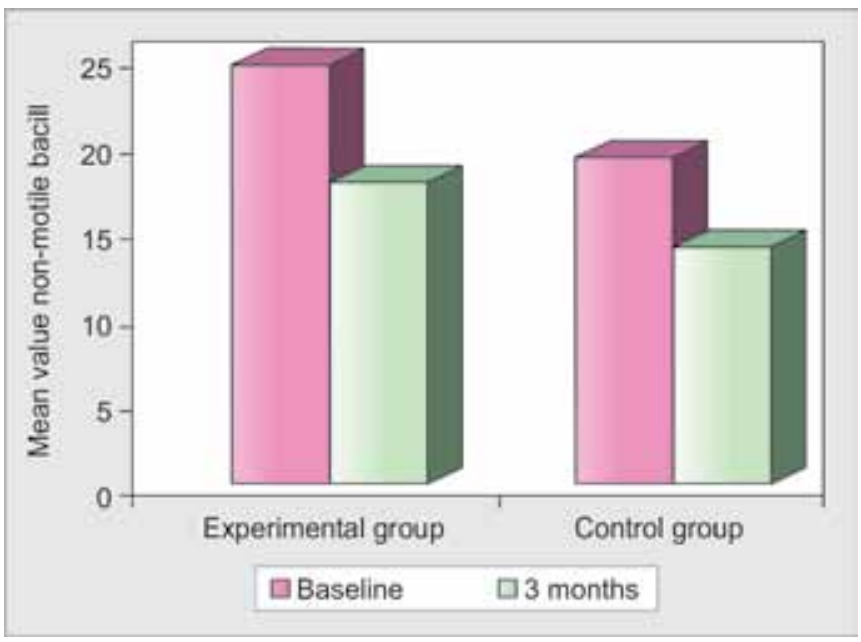

Graph 4: Mean values of nonmotile bacilli

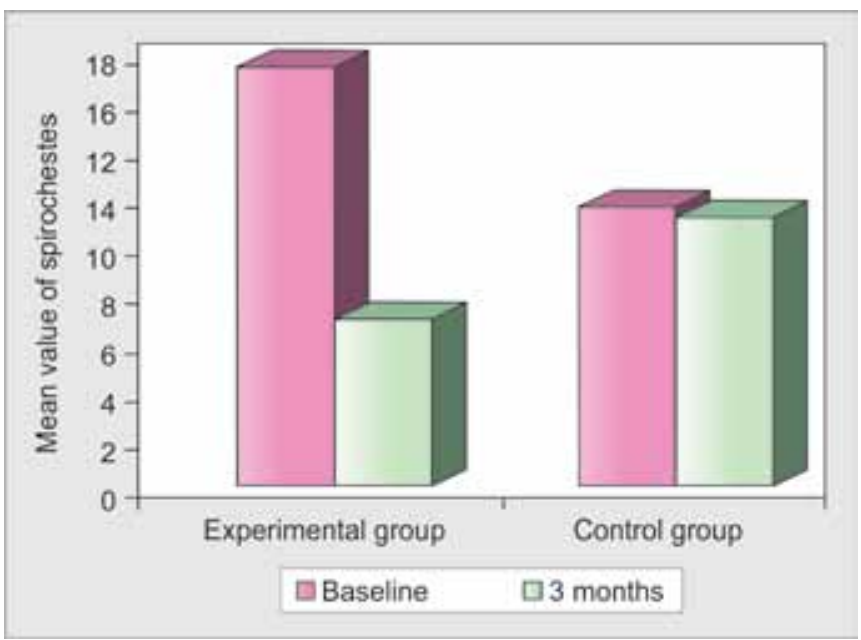

Graph 5: Mean values of spirochetes 


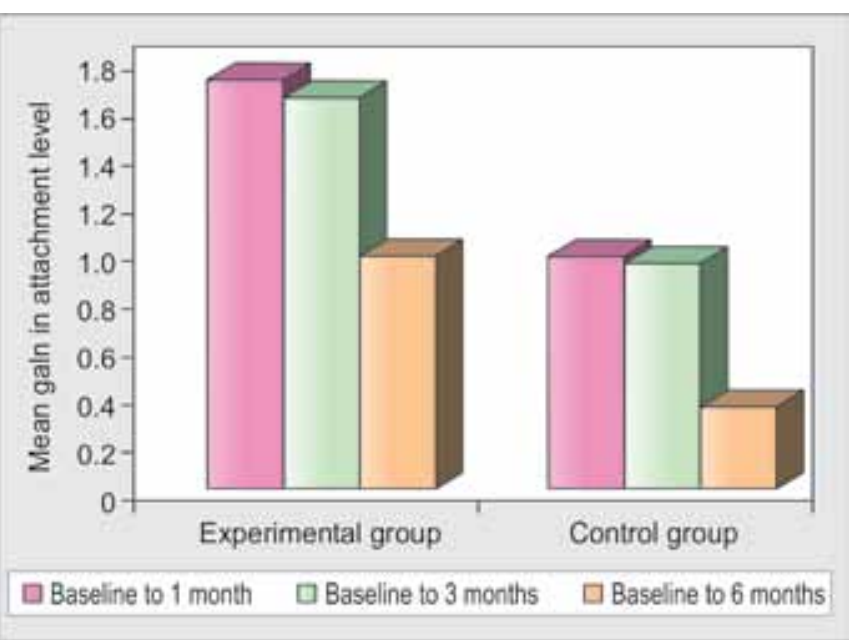

Graph 6: Mean gain in attachment levels at different time intervals

\section{Gain in Attachment Level}

When experimental and control group sites were compared the mean gain in attachment level in experimental group was $1.00 \pm 0.7 \mathrm{~mm}$ at 180 th days. For control group the mean gain in attachment level was $0.36 \pm 0.4 \mathrm{~mm}$ at 180 th days. When inter group comparisons was done between experimental and control group the gain in attachment level showed gain which was statistically significant $(\mathrm{p}<0.05)$.

\section{Microbiological Analysis}

\section{Cocci}

When control sites and experimental sites were evaluated for the mean number of cocci intragroup comparison showed that there was an increase in cocci in experimental group as well as control group from baseline to 90th days. This was found to be not statistically significant $p>0.23$. Intergroup comparison showed no statistical significance in both experimental as well as control group.

Intragroup comparison of control sites and experimental sites showed there was reduction in nonmotile bacilli in both experimental as well as control group from baseline to 90th days. This was not found to be statistically significant for both the groups ( $p>0.30, p>0.12$ respectively). Inter group comparison showed no statistical significance $p>0.4$. Intragroup comparison showed that when nonmotile bacilli and cocci were combined both experimental and control groups showed no statistical significance $(p>0.12,>$ 0.37 respectively).

\section{Motile Bacilli}

Intragroup comparison of control sites and experimental sites showed the re was reduction in motile bacilli in both experimental as well as control group from baseline to 90th days. This was not found to be statistically significant for both the groups ( $p>0.07, p>0.12$ respectively). Intergroup comparison showed no statistical significance $p>0.4$. Intragroup comparison showed that when motile bacilli and spirochetes were combined it showed statistical significance in the experimental group $\mathrm{p}<0.03$. There was no statistical significance for control group $\mathrm{p}>0.23$.

\section{Spirochetes}

Intragroup comparison showed there was reduction in number of spirochetes in both experimental as well as control group from baseline to 90th days. This was found to be statistically significant for experimental group $p<0.02$. For control group, it was not statistically significant $\mathrm{p}>0.17$. Statistical analysis of inter group comparison showed no statistical significance $p>0.07$ in both experimental as well as control group.

\section{DISCUSSION}

The major factor in the etiology of periodontal disease is bacterial plaque associated biofilm. Hence the primary goal of conventional periodontal therapy is the alteration of subgingival biofilm on periodontal disease sites (Garrett et al). ${ }^{3}$ Though SRP is considered to be gold standard for the prevention of periodontal disease, it is not found to be successful at all treated sites. Though not all factors influencing failure of nonsurgical therapy are known, deep periodontal pockets Rabbani et $\mathrm{al}^{8}$ and furcation involvement Fleischer et $\mathrm{al}^{9}$ are some of them. In this attempt, various systemic and local anti-microbial agents came to existence in order to provide additional benefits (Goodson et al). ${ }^{10}$ Various authors like Kornman, ${ }^{11}$ Greenstein ${ }^{12}$ have stated that locally delivered antimicrobials offers many more advantages as compared to systemic therapy, including maintaining maximum concentration of the drug at the site of action for prolonged periods of time with minimal side effects.

Goodson et $\mathrm{al}^{10}$ pointed out that successful control of the periodontal microflora requires delivery of an antimicrobial agent that should reach the depth of a periodontal pocket and in surrounding tissues and maintain minimum effective concentrations for sufficient duration to produce the therapeutic effect. In the present study $10 \%$ Doxycycline Hyclate gel from a biodegradable, controlledrelease delivery system Atridox was chosen because, it has a broad spectrum of bacteriostatic action and also ability to maintain effective concentration at target site, low systemic toxicity and its proven clinical efficacy. Polson et $\mathrm{a} \mathrm{l}^{13}$ Wennstrom et al,,${ }^{14}$ Luciano Machion et $\mathrm{a} 1{ }^{15}$ at present, however few studies are available showing additional microbial benefits of the subgingival application of this drug in combination with root planing. Hence an attempt 
was made in the present study to compare the efficacy of combined therapy (i.e SRP with drug application) with SRP alone in reducing the microbial counts.

In this study parallel-design was followed in order to avoid any cross-over effect of the test drug Polson et al. ${ }^{13}$ As reported by Lowenguth et $\mathrm{al}^{16}$ that the reduction in subgingival microbiota following any treatment would be within a period of 90 days following which there will be gradual rise in the number of organisms hence microbial parameter for our study was performed at baseline and 90th days. Microbial sampling was performed using 3 sterile endodontic paper points as suggested by Kornman ${ }^{11}$ and all the samples were transported to the laboratory immediately. The test drug was prepared following the manufacturer's instructions and applied in all the selected test sites. Periodontal dressing was placed after the drug application / treatment in order to maintain the uniform retention of the drug Stolleret al. ${ }^{17}$ In our study no adverse effects of the drug was reported. In contrast, Garrett et $\mathrm{al}^{3}$ reported adverse effect in $<1 \%$ of the subject. Both the groups exhibited a significant improvement in periodontal status over a period of 180 days as compared to baseline. Intragroup observations for the gingival index showed significant reduction in gingival score from baseline to 180 days. This is similar to the studies conducted by Heijl et al, ${ }^{18}$ Friesen et al. ${ }^{19}$ While comparing the gingival score between the two groups with values respectively at baseline to 180 days, there was no statistical significance observed at any interval. This could be attributed to the Hawthorne effect, which states that the fact that individuals in a clinical study will often encourage them to perform better oral hygiene Goodson et al. ${ }^{20}$ When gingival index and plaque index of only test sites were measured, experimental group produced greater reduction in gingival index scores than the control group whereas plaque index scores did not differ between the two groups.

From this observation, it can be interpreted that, there was no cross-over effect of $10 \%$ Doxycycline gel because the test sites showed greater reduction in gingival inflammation. Hence split-mouth design can be used as proposed by Goodson et $\mathrm{al}^{21}$ in the clinical trials to assess the efficacy of $10 \% \mathrm{DH}$ gel, which was initially in contradiction to our decision of employing a parallel-study design.

Intragroup observation for gingival bleeding index, showed significant reductions from baseline to 180th days for both the groups. This is in consistent with the findings of Newman et al. ${ }^{22}$ Intergroup comparison between experimental as well as control group showed statistically significant reduction in bleeding scores at 180 days from baseline in experimental group.

The pocket depth reduction for both the groups between baseline and 180 days was found to be statistically significant.
Similar observations were made by Luciano Machion et al. ${ }^{15}$ In both the groups there was significant gain in attachment level as compared to baseline. When intergroup comparison was made experimental group exhibited more gain in attachment level which was statistically Goodson et al. ${ }^{20}$ Maximum reduction in probing depth and gain in attachment level was seen at 30 days and 90 days.

A quantitative analysis of the pathogenic bacteria present in the subgingival ecosystem is an ideal marker to evaluate the effectiveness of nonsurgical mechanical periodontal therapy. Listgarten et al, ${ }^{23}$ claimed that nonmotile bacteria are associated with periodontal health whereas motile microorganisms with the disease Ojima et al. ${ }^{24}$ In other words, decrease of total motile microorganisms to very low levels represents a remarkable indicator for the success of periodontal treatment Lavanchy et al. ${ }^{25}$

In the present study sites were evaluated by dark field microscope for percent age of spirochetes, motile bacilli and cocci, concomitantly increase in coccoid cell counts was not statistically significant. Intragroup comparisons for spirochetes and coccoid cell count revealed significant reduction in spirochete count and proportionate increase in the coccoid cell count in the experimental groups. Whereas for the control group this reduction was not statistically significant. Inter groups comparison revealed no statistical difference in the reduction of spirochetes and motile bacilli. This is in agreement with Greenwell et al, ${ }^{26}$ who opined that, subgingival instrumentation induced marked shifts in the subgingival microflora with significant reduction in the motile, gram negative organisms. The results of the this study demonstrated that the local drug delivery of $10 \%$ Doxycycline Hyclate gel is significantly advantageous both clinically and microbiologically over routine SRP especially when used as an adjunct to SRP in the nonsurgical management of pockets with 6 to $7 \mathrm{~mm}$ depth. No attempt was made to assess the efficacy of $10 \%$ Doxycycline Hyclate gel against main periodontopathic anaerobic bacteria. Additional randomized, controlled studies are needed to demonstrate the efficacy of this drug in sites with refractory or recurrent disease or specific locations.

\section{CONCLUSION}

The results of this study demonstrated that subjects in both the groups exhibited a significant improvement in periodontal status from baseline. The sites in the experimental group exhibited better results as compared to the control group. The reduction in spirochetes count is more significant in the experimental group. Combination therapy of SRP and $10 \%$ doxycycline hyclate gel clearly demonstrates the superiority at all the levels suggesting that the drug can play a significantly advantages role as an added therapeutic agent in the management of periodontal diseases. 
Further studies with larger samples are required to establish the effects of $10 \%$ doxycycline hyclate gel in different periodontitis cases.

\section{REFERENCES}

1. Kulkarni GV, Lee WK, Aitken S, Birek P, McCulloch CA. A randomized placebo - controlled trial of doxycycline: effect on the microflora of recurrent periodontitis lesions in high risk patients. J Periodontol 1991;62:197-202.

2. Polson AM, Southard GL, Dunn RL, Yewey GL, Godowski KC, Polson AP, et al. Periodontal pocket treatment in beagle dogs using subgingival doxycycline from a biodegradable system. Initial clinical responses. J Periodontol 1996;67:1176-1184.

3. Garrett S, Johnson L, Drisko CH, Adams DF, Bandt C, Beiswanger B, et al. Two 9 months multicenter studies evaluating locally delivered doxycycline hyclate $8.5 \%$, placebo control oral hygiene and scaling and root planing in the treatment of periodontitis. J Periodontol 1999;70:490-503.

4. Walker KC, Godowski L, Borden L, Lennon J, Nangó S, Stone $\mathrm{C}$, et al. The effects of sustained release doxycycline on the anaerobic flora and antibiotic resistant patterns in subgingival plaque and saliva. J Periodontol 2000;71:768-774.

5. Sillness J, Loe H. Periodontal diseases in pregnancy (2) correlation between oral hygiene and periodontal condition. ActaOdontol Scan 1964;24:747-759.

6. Ainamo J, Bay I. Problems and proposals for recording gingivitis and plaque. Int Dent J 1975;25:229-235.

7. Lobene RR, Weatherford T, Rose NM, Lamm RA, Menaker L. A modified gingival index for use in clinical trials. Clin Prev Dent 1986;8:3-6.

8. Rabbani GM, Ash MM, Caffesse RG. The effectiveness of subgingival scaling and root planing in calculus removal. J Periodontol 1981;52:119-123.

9. Fleischer HC, Mellonig JT, Brayer WK, Gray JL, Barnett JD. Scaling and root planing efficacy in multirooted teeth. J Periodontol 1989;60:402-409.

10. Goodson JM, Offenbacher S, Farr DH, et al. Periodontal disease treatment by local drug delivery. J Periodontol 1985;56:265-272.

11. Kornman KS. Controlled - release local delivery antimicrobials in periodontics: prospects for the future. J Periodontol 1993;64: 782-791.

12. Greenstein G, Polson AM. The role of local drug delivery in the management of periodontal diseases: a comprehensive review. J Periodontol 1998;69:507-520.

13. Polson AM, Garrett S, Stoller N, et al. Multicenter comparative evaluation of subgingivally delivered sanguinarine and doxycycline in the treatment of periodontitis. II. Clinical results. J Periodontol 1997;68:119-126.

14. Wennstrom JL, Tomasi C. Locally delivered doxycycline improves the healing following nonsurgical periodontal therapy in smokers'. J Clin Periodontol 2004;31:589-595.

15. Luciano Machion, et al. Locally delivered Doxycycline as an adjunctive therapy to scaling and root planing in the treatment of smokers: a clinical study. J Periodontol 2004;75:464-469.

16. Lowenguth RA, Chin I, Caton JG, Cobb CM, Drisko CL, Killoy WJ, Michalowicz BS, Pihlstrom BL, Goodson JM. Evaluation of periodontal treatments using controlled-release tetracycline fibers: microbiological response. J Periodontol 1995;66: 700-707.

17. Stoller NH, Johnson LR, Trapnell S, Harrold CQ, Garrett S. The pharmacokinetic profile of a biodegradable controlledrelease delivery system containing doxycycline compared to systemically delivered doxycycline in gingival crevicular fluid, saliva and serum. J Periodontol 1998;69:1085-1091.

18. Heijl L, Dachen G, Sundin Y, Wenader A, Goodson JM. A 4 quadrant comparative study of periodontal treatment using tetracycline containing drug delivery fibers and scaling. J Clin Periodontol 1991;18:111-116.

19. Friesen LR, Williams KB, Krause LS, Killoy WJ. Controlled local delivery of tetracycline with polymer strips in the treatment of periodontitis. J Periodontol 2002 Jan;73(1):13-19.

20. Goodson JM. Discussion: design issue specific to studies of periodontitis. J Periodontol Res 1992(spec issue);27: 412-416.

21. Goodson JM, Cugini MA, Kent RL, Armitage GC. Multicentre evaluation of Tetracycline fiber therapy. II clinical responses. J Periodont Res 1991;26:371-379.

22. Newman MG, Kornman KS, Doherty FM. A 6-month multicenter evaluation of adjunctive tetracycline fiber therapy used in conjunction with scaling and root planing in maintenance patients: clinical results. J Periodontol 1994;65:685-691.

23. Lisgarten MA, Hellden L. Relative distribution of bacteria at clinically healthy and periodontally disease sites in human. J Clin Periodontol 1978;5:115-132.

24. Ojima M, Tamagawa WH, Nagata H. Relation of motility of subgingival microflora as a clinical parameter to periodontal disease status in human subjects. J Clin Periodontol 2000;27: 405-410.

25. Lavanchy DL, Bickel, Baehni PC. The effect of plaque control after scaling and root planing on the subgingival microflora in human periodontitis. J Clin Periodontol 1987;14:295-299.

26. Greenwell H, Bissada NF. Variations in subgingival microflora from healthy and intervention sites using probing depth and bacteriologic identification criteria. J Periodontol 1984;55: 391-397.

\section{ABOUT THE AUTHORS}

\section{Shabeer Ahamed (Corresponding Author)}

Associate Professor, Department of Periodontics, Malabar Dental College, Edapal, Kerala, India, Phone: 9746074655, e-mail: drshabeer9582@gmail.com

\section{Md Jalaluddin}

Reader, Department of Periodontics and Oral Implantology, Kalinga Institute of Dental Sciences, Bhubaneswar-24, Odisha, India

\section{Imran Khalid}

Reader, Department of Oral and Maxillofacial Surgery, MGM Dental College, Navi Mumbai, Maharashtra, India

\section{Ninad Moon}

Professor and Head, Department of Periodontics, RKDF Dental College and Research Centre, Bhopal, Madhya Pradesh, India

\section{TK Shafi}

Reader, Department of Periodontics, Kannur Dental College, Kannur, Kerala, India

\section{Fareedi Mukram Ali}

Reader, Department of Oral and Maxillofacial Surgery, SMBT Dental College, Sangamner Taluka, Maharashtra, India 